1 Departamento de Nutrição, Faculdade de Saúde Pública, Universidade de São Paulo (USP), São Paulo, SP, Brasil ${ }^{2}$ Departamento de Medicina Preventiva, Universidade Federal de São Paulo (Unifesp), São Paulo, SP, Brasil

${ }^{3}$ Ver apêndice

Trabalho realizado no Departamento de Nutrição, Faculdade de Saúde Pública, Universidade de São Paulo (USP), São Paulo, SP, Brasil
Correspondência para: Maria F Cristofoletti

Praça Marechal Deodoro, 397, ap. 66 01150-011 - São Paulo, SP, Brasil fcristofoletti@hotmail.com

Recebido em 29/Abr/2013 Aceito em 28/Jun/2013

\section{Associação entre consumo de alimentos embutidos e obesidade em um estudo de base populacional de nipo-brasileiros}

\author{
Association of processed meat intake and obesity in a \\ population-based study of Japanese-Brazilians
}

Maria F. Cristofoletti', Suely G. A. Gimeno², Sandra R. G. Ferreira', Marly A. Cardoso', Japanese-Brazilian Diabetes Study Group ${ }^{3}$

\section{RESUMO}

Objetivo: Investigar a associação entre consumo de alimentos embutidos e obesidade generalizada, abdominal e generalizada com abdominal em nipo-brasileiros de Bauru, SP. Sujeitos e métodos: Quatrocentos e quarenta e três mulheres e 329 homens nipo-brasileiros não miscigenados ( $\geq 30$ anos) foram avaliados em estudo transversal de base populacional. Para o diagnóstico de obesidade, foram empregados os critérios da Organização Mundial da Saúde para asiáticos. A ingestão de alimentos foi avaliada por meio de questionário de frequência alimentar validado. A ingestão foi estratificada em terços para análise. Resultados: Nos homens, a ingestão de colesterol e alimentos embutidos mostrou-se positivamente associada à obesidade generalizada com abdominal quando o primeiro terço de ingestão foi comparado ao último, após ajustes (OR 2,97; IC95\% 1,13-7,78). Em mulheres, somente o grupo das carnes vermelhas associou-se à obesidade geral com abdominal após ajustes (OR 0,47; IC95\% 0,23-0,96). Conclusão: Ingestão elevada de alimentos embutidos associou-se à obesidade generalizada com adiposidade abdominal em homens nipo-brasileiros, mas não em mulheres. Arq Bras Endocrinol Metab. 2013;57(6):464-72

Descritores

Obesidade abdominal; obesidade; nipo-brasileiros; gorduras; consumo alimentar

\section{ABSTRACT}

Objective: The aim of this study was to investigate the association between the consumption of processed meat with overall, abdominal, and overall with abdominal obesity in a Japanese-Brazilian population, which is known to be at cardiometabolic risk. Subjects and methods: A total of 329 men and 443 women aged $\geq 30$ years were evaluated in a cross-sectional population-based survey. Diagnosis of overall obesity and abdominal obesity were based on the World Health Organization (WHO) criteria for Asians. Food intake was assessed by a validated food frequency questionaire. Results: In men, processed meat intake was positively associated with overall with abdominal obesity (OR $2.97 ; 95 \% \mathrm{Cl} 1.13-7.78$ ) after adjustment. In women, only the red meat group was associated with overall with abdominal obesity after adjustment (OR 0.47 , $95 \% \mathrm{Cl}$ 0.23-0.96). Conclusion: Our results showed that high intakes of processed meats were associated with overall with abdominal obesity in male Japanese-Brazilians, but not in females. Arq Bras Endocrinol Metab. 2013;57(6):464-72

\section{Keywords}

Abdominal obesity; obesity; Japanese-Brazilians; fats; dietary intake 


\section{INTRODUÇÃO}

$\mathrm{E}$ studos em diferentes populações no mundo descrevem o papel do estilo de vida, especialmente fatores dietéticos, na etiologia da obesidade (1). Evidências encontradas na literatura divergem sobre o papel das gorduras e de alimentos ricos em gorduras, como o grupo das carnes, no aumento dos depósitos de gordura corporal, principalmente de localização abdominal. Três estudos transversais encontraram correlação positiva entre o perímetro de cintura (PC) e consumo de gorduras totais (2-4). Quanto aos estudos longitudinais, os resultados são inconclusivos. Um estudo verificou correlação negativa entre ingestão de gorduras totais e o PC (5). Outras evidências oriundas de um estudo longitudinal sugerem que o acúmulo de gordura na região abdominal é fortemente associado às frações de gordura consumida, sobretudo a gordura trans (6). Entre os grupos de alimentos fontes de gordura, como as carnes vermelhas e os embutidos, os resultados derivados de estudos transversais e longitudinais parecem apontar para um possível risco para a obesidade abdominal e geral $(7,8)$.

Há evidências de maior incidência de diabetes tipo 2 e doenças associadas em imigrantes japoneses que adquiriam hábitos ocidentais (9). No Brasil, a população nipo-brasileira do Município de Bauru, SP, apresenta uma das maiores prevalências de distúrbios da homeostase glicêmica no mundo (10). O aumento da resistência à insulina em populações migrantes relaciona-se à obesidade abdominal, especialmente à gordura visceral (11). Em nipo-brasileiros de Bauru, Lerário e cols. (12) observaram que a alta prevalência de diabetes tipo 2 associava-se ao ganho de peso corporal e à obesidade abdominal. Tal associação tem sido atribuída à produção de adipocitocinas pró-inflamatórias que contribuem para deteriorar a sensibilidade à insulina (13).

A respeito do papel das gorduras na gênese do diabetes tipo 2 e doenças associadas nesta população, um maior consumo de gordura e carnes vermelhas em nipo-brasileiros associou-se à síndrome metabólica $(14,15)$. Associação inversa entre consumo total de gordura saturada e doença macrovascular (16) também foi descrita, observando-se, por outro lado, associação inversa com o consumo de fibra dos grãos.

No presente estudo, investiga-se na população nipo-brasileira de Bauru, SP, a existência de associação entre alimentos embutidos com obesidade, considerando-se a distribuição da adiposidade, generalizada ou central.

\section{INDIVÍDUOS E MÉTODOS}

\section{População de estudo}

Censo populacional de 1997 identificou 1.751 nipo-brasileiros em Bauru, SP, de primeira e segunda geração, não miscigenados, com idade $\geq 30$ anos. Em 1999 e 2000, foram coletados dados de 1.330 indivíduos que assinaram o Termo de Consentimento Livre e Esclarecido para participar deste estudo, aprovado pelo Comitê de Ética da Universidade Federal de São Paulo. Os motivos para a não participação foram: óbito (antes ou durante o trabalho de campo, $\mathrm{n}=94$ ), mudança de endereço $(n=57)$ e recusa $(n=270)$. Para a presente análise, foram excluídos 558 indivíduos $(41,9 \%)$, dos quais $47(3,5 \%)$ não completaram todas as etapas da avaliação nutricional, 148 (11,1\%) com diagnóstico prévio de diabetes mellitus (DM), $72(5,4 \%)$ com intolerância à glicose, $9(0,7 \%)$ por não completarem o teste oral de tolerância à glicose, $267(20,0 \%)$ com diagnóstico de diabetes mellitus tipo 2 (DM) em 2000, 15 (1,1\%) com valores de proteína $\mathrm{C}$ reativa (PCR) iguais ou superiores a $1 \mathrm{mg} / \mathrm{dL}$. Participantes com $\mathrm{DM}$, intolerância à glicose e PCR elevada não foram incluídos na presente análise devido à possibilidade de terem modificado o padrão de consumo alimentar ou possível estado inflamatório agudo (18). Dessa forma, para os propósitos deste estudo, o universo amostral incluiu 772 participantes ( 329 homens e 443 mulheres) (44,1\% do censo de 1997).

\section{Coleta de dados e avaliação clínica}

Em entrevista domiciliar, foram aplicados questionários estruturados contemplando dados sociodemográficos e de saúde. Atividade física (sedentário e leve ou moderada e intensa), tabagismo (atual, nunca fumou ou ex-fumantes) e consumo de bebidas alcoólicas (ausente, 0,2-54 ou > 54 gramas/dia) foram classificados em categorias.

A pressão arterial (PA) foi aferida sentada, após repouso, por esfigmomanômetro automático (Omron, modelo HEM-712C, Omron Health Care, Inc, USA). Considerou-se o valor médio das duas últimas medidas. Hipertensão arterial (HA) foi definida quando o indivíduo apresentasse PA sistólica ou diastólica $\geq 140 / 90$ $\mathrm{mmHg}(19)$ ou usasse medicamentos anti-hipertensivos.

Peso, altura e perímetro da cintura foram medidos com os indivíduos na posição ereta, usando o mínimo de roupas possíveis e sem sapatos. O perímetro da cintura foi medido durante a expiração na altura da 
cicatriz umbilical. Os pontos de corte utilizado foram os da Organização Mundial da Saúde (OMS) para asiáticos, ou seja, $\mathrm{PC} \geq 90 \mathrm{~cm}$ para homens e $\geq 80 \mathrm{~cm}$ para mulheres (20). O índice de massa corporal (IMC) foi calculado pelo peso (em quilos) dividido pela altura (em metros) ao quadrado (1), definindo-se obesidade "generalizada" pelo IMC $\geq 25 \mathrm{~kg} / \mathrm{m}^{2}(20)$. Obesidade geral na presença de abdominal foi definida quando o indivíduo apresentasse simultaneamente obesidade generalizada e abdominal.

\section{Avaliação do consumo alimentar}

Dados dietéticos foram obtidos a partir de um questionário quantitativo de frequência alimentar (QQFA) desenvolvido e validado para a população nipo-brasileira (21). Os indivíduos responderam sobre seu consumo habitual de alimentos ou grupos alimentares no último ano, informando sobre frequência de consumo em unidades de tempo e o tamanho usual da porção. Questões relacionadas ao uso de temperos, frequência da ingestão de gorduras visíveis e tipo de gordura utilizada no preparo dos alimentos também foram incluídas. A análise das dietas foi realizada por meio do Dietsys 4.0 (National Cancer Institute, Bethesda, Maryland, USA) com dupla digitação e análise de consistência. A composição química dos alimentos foi baseada em tabelas oficiais do Brasil, Japão e Estados Unidos, conforme previamente descrito (22).

Os teores de ácidos graxos trans dos alimentos do QQFA foram obtidos em tabelas nacionais e internacionais $(23,24)$. Para alguns alimentos e preparações nacionais não identificados nas tabelas publicadas, utilizou-se o teor de ácidos graxos trans de alimento de composição química e processamentos semelhantes (25). As variáveis dietéticas de interesse foram calorias totais, gordura total, gordura total em relação ao valor calórico total (\%VCT), colesterol, gordura saturada, gordura trans, ácido graxo oleico, ácido graxo linoleico, embutidos (presunto, mortadela, linguiça e salsicha); carnes vermelhas (carnes vermelhas em geral, bovina e suína); aves (aves em geral, frango e peru); pescados e frutos do mar (peixes em geral, sashimi [peixe cru], chikuwa [embutido à base de peixe] e camarão).

\section{Indicadores bioquímicos}

Foram coletadas amostras sanguíneas em jejum para determinação de glicose, insulina, perfil lipídico e PCR. A seguir, os participantes foram submetidos ao teste oral de tolerância à glicose com $75 \mathrm{~g}$. Após duas horas da sobrecarga, foi realizada nova coleta para determinação de glicose e insulina. A glicose plasmática foi determinada pelo método da glicose-oxidase (26). Para cada série de dosagem de glicose, foram obtidas três amostras controles, cuja variação dos valores foi inferior a 5\%. Para fins deste estudo, adotou-se a classificação da homeostase glicêmica segundo a OMS (27), ou seja: normal (glicemia de jejum < $110 \mathrm{mg} / \mathrm{dL}$ e glicemia pós-carga de glicose $<140 \mathrm{mg} / \mathrm{dL}$ ); glicemia de jejum alterada - GJA (glicose de jejum entre $110 \mathrm{mg} / \mathrm{dL}$ e $126 \mathrm{mg} / \mathrm{dL}$ com glicemia de 2 horas < $140 \mathrm{mg} / \mathrm{dL}$ ); tolerância de glicose diminuída - TGD (glicemia de jejum $<126 \mathrm{mg} / \mathrm{dL}$ e após sobrecarga entre $140 \mathrm{mg} / \mathrm{dL}$ e $200 \mathrm{mg} / \mathrm{dL}$ ) e DM (glicemia de jejum $\geq 126 \mathrm{mg} / \mathrm{dL}$ e/ou pós-sobrecarga $\geq 200 \mathrm{mg} / \mathrm{dL}$ ). A insulina foi determinada por método imunofluorimétrico baseado em anticorpo monoclonal (Auto Delfia, Perkin Elmer Life Science Inc, Norton, USA) (28). O HOMA-IR (bomeostatic model assessment) foi calculado para estimar a resistência a insulina [HOMA-IR = glicose em jejum $(\mathrm{mmol} / \mathrm{L}) \mathrm{x}$ insulina em jejum $(\mathrm{mU} / \mathrm{L}) / 22,5](29)$.

Os lípides séricos foram determinados por métodos enzimáticos. O diagnóstico de dislipidemia foi definido quando o indivíduo apresentasse colesterol total $\geq$ $200 \mathrm{mg} / \mathrm{dL}$ ou LDL-c $\geq 130 \mathrm{mg} / \mathrm{dL}$ ou triglicérides $\geq$ $150 \mathrm{mg} / \mathrm{dL}$ ou HDL-colesterol (HDL-c) $\leq 40 \mathrm{mg} / \mathrm{dL}$, conforme os critérios do National Cholesterol Education Program (NCEP) ou em uso de medicamentos antilipêmicos (30). Níveis de proteína $\mathrm{C}$ reativa (PCR) foram determinados por quimiluminescência (Immulite High Sensitivity CRP Assay; DPC, Los Angeles, CA, USA).

\section{Análise estatística}

Os desfechos foram obesidade abdominal (medida pelo PC), obesidade generalizada (medida pelo IMC) e obesidade generalizada na presença de abdominal. As variáveis dietéticas consideradas de exposição foram gordura total, gordura saturada, ácidos graxos trans, ácido graxo oleico e ácido graxo linoleico, colesterol, além dos grupos alimentares: embutidos, carnes vermelhas, aves e peixe e frutos do mar.

As análises foram realizadas com o auxílio do SPSS versão 12.0 (SPSS Inc., Chicago, IL, USA, 2003), com o valor de $\mathrm{p}<0,05$. Uma subamostra (657 indivíduos: 378 mulheres e 279 homens) foi utilizada em análises complementares com o desfecho: obesidade generalizada na presença de obesidade abdominal $(\mathrm{n}=210)$, excluindo-se 115 indivíduos que apresentavam so- 
mente obesidade generalizada $(n=61)$ ou aqueles que apresentavam apenas obesidade abdominal $(\mathrm{n}=54)$. Frequências relativas e absolutas, média, desvio-padrão (DP), mediana e intervalo interquartil das variáveis sociodemográficas, bioquímicas, dietéticas (dados brutos) e antropométricas foram calculados segundo: presença de obesidade generalizada, obesidade abdominal e obesidade generalizada na presença de abdominal. As variáveis bioquímicas e de consumo alimentar sofreram transformação logarítmica antes das análises estatísticas. Os nutrientes da dieta foram ajustados pelas calorias totais pelo método residual. $\mathrm{O}$ ajuste foi realizado por regressão linear simples, com o nutriente como variável dependente e as calorias totais como independente. $\mathrm{O}$ nutriente ajustado foi o resultado da soma do resíduo, obtido pela regressão linear simples, com o valor esperado para o consumo do nutriente para indivíduos com ingestão habitual igual à média de calorias totais para a população (31). Para comparação entre variáveis contínuas e proporções, foram empregados teste $t$ de Student ou ANOVA ou U de Mann-Whitney e Quiquadrado, respectivamente. As variáveis dietéticas foram categorizadas em terços para a análise de associação com os desfechos de interesse em modelos de regressão logística estratificados por sexo. A seleção das variáveis dietéticas para os modelos múltiplos finais seguiu critérios estatísticos e plausibilidade biológica. Estimativa da odds ratio (OR) e do respectivo intervalo de confiança foi calculado para os três desfechos (obesidade abdominal, obesidade generalizada e obesidade generalizada na presença de abdominal). $\mathrm{O}$ menor tercil de ingestão considerou-se a categoria de referência. Testou-se a tendência de variação linear no risco ( $\mathrm{p}$ de tendência) com os valores medianos do intervalo de cada terço das variáveis de exposição de interesse como valores de uma variável contínua. O modelo inicial foi ajustado para a idade (em anos). Em seguida, o modelo sofreu ajuste para as covariáveis: geração (primeira e segunda), fumo (nunca, ex-fumante, fumante atual), hipertensão (sim, não ou uso de medicamentos), dislipidemia (sim, não ou uso de medicamentos), atividade física no trabalho ( $\operatorname{sim}$, não) e consumo de bebidas alcoólicas (nunca, 0,2 a 54 gramas/dia, > 54 gramas/dia). Por fim, o modelo múltiplo foi adicionalmente ajustado para calorias totais (kcal) e HOMA-IR. Outros modelos foram calculados com ajuste adicional para IMC (quando o desfecho foi obesidade abdominal) ou PC (quando o desfecho foi obesidade generalizada).

\section{RESULTADOS}

As características gerais dos participantes segundo gênero são descritas na tabela 1 . Os homens apresentaram maior escolaridade, havendo ainda maior proporção de casados, tabagistas, consumidores de bebidas alcoólicas, dislipidêmicos e com alterações da tolerância à glicose. A frequência de mulheres sedentárias ou com atividades leves foi maior que nos homens.

Estratificando-se segundo presença de obesidade generalizada, abdominal e generalizada na presença de abdominal (Tabela 2), observa-se, conforme esperado, que indivíduos com obesidade generalizada na presença de abdominal apresentaram valores médios maiores de IMC e PC, além de maior proporção de portadores de TGD e PA elevada PA e com maior mediana do HOMA-IR.

Em homens, observou-se que o maior consumo de ácido graxo oleico e grupo dos embutidos ajustados para idade associou-se à obesidade abdominal. Após ajuste adicional para covariáveis, o maior consumo de ácido graxo oleico foi associado à obesidade abdominal. Em relação ao grupo dos embutidos, o consumo no último tercil foi associado à obesidade abdominal após ajustes. Contudo, após ajuste adicional para IMC, a associação perdeu significância. Quando comparado apenas o último terço com o primeiro, houve tendência de aumento do risco com maior nível de ingestão dos embutidos (Tabela 3).

$\mathrm{Na}$ população masculina, houve associação entre obesidade generalizada com abdominal e o maior consumo de embutidos, aves, pescados e frutos do mar após ajuste para idade quando comparado o maior com o menor terço de ingestão. O maior consumo de aves e pescados e frutos do mar foi associado à obesidade generalizada com abdominal após ajustes; porém, essas associações não persistiram com ajuste adicional para HOMA-IR (Tabela 3).

Considerando ainda a população masculina, observou-se associação positiva entre obesidade generalizada com abdominal e a maior ingestão de colesterol e embutidos, quando comparado o primeiro com o último terço de ingestão após ajustes. Além disso, o consumo de embutidos apresentou relação dose-resposta com tendência linear para aumento do risco com seu maior consumo (Tabela 3 ).

Em mulheres, somente o grupo das carnes vermelhas associou-se à obesidade generalizada na presença de abdominal após ajustes $\left(\mathrm{OR}_{3^{\circ} \text { tercil }} 0,47\right.$; IC95\% 0,23$0,96)$. 
Tabela 1. Características da população nipo-brasileira segundo gênero

\begin{tabular}{|c|c|c|c|c|c|}
\hline \multirow[b]{2}{*}{ Geração (n \%) } & \multicolumn{2}{|c|}{$\begin{array}{c}\text { Homens } \\
(\mathrm{n}=329)\end{array}$} & \multicolumn{2}{|c|}{$\begin{array}{l}\text { Mulheres } \\
(n=443)\end{array}$} & \multirow[t]{2}{*}{$\mathbf{P}$} \\
\hline & & & & & \\
\hline Primeira & 372 & 48,2 & 400 & 51,8 & 0,319 \\
\hline Segunda & 320 & 41,4 & 43 & 58,6 & \\
\hline Idade (anos) & \multicolumn{2}{|c|}{$55,1(13,0)$} & \multicolumn{2}{|c|}{$55,2(12,4)$} & 0,862 \\
\hline \multicolumn{6}{|l|}{ Escolaridade $(\mathrm{n} \%)^{*}$} \\
\hline$<1$ ano & 279 & $\begin{array}{l}36,1 \\
39,0 \\
53,6\end{array}$ & 493 & $\begin{array}{l}63,9 \\
61,0 \\
46,4\end{array}$ & 0,022 \\
\hline $1-8$ anos & 301 & 39,0 & 471 & 61,0 & \\
\hline$\geq 8$ anos & 414 & 53,6 & 358 & 46,4 & \\
\hline \multicolumn{6}{|l|}{ Estado civil $(\mathrm{n} \%)^{*}$} \\
\hline Casado & 380 & $\begin{array}{l}49,2 \\
25,9 \\
18,8\end{array}$ & 392 & $\begin{array}{l}50,8 \\
74,1 \\
81,8\end{array}$ & 0,000 \\
\hline Solteiro & 200 & 25,9 & 572 & 74,1 & \\
\hline Viúvo/divorciado & 145 & 18,8 & 627 & 81,2 & \\
\hline \multicolumn{6}{|l|}{ Atividade física no trabalho $(\mathrm{n} \%)^{*}$} \\
\hline Sedentária ou leve & 284 & $\begin{array}{l}36,8 \\
60,2\end{array}$ & 408 & $\begin{array}{l}63,2 \\
39,8\end{array}$ & 0,001 \\
\hline Moderada ou intensa & 465 & 60,2 & 307 & 39,8 & \\
\hline \multicolumn{6}{|l|}{ Tabagismo $(\mathrm{n} \%)^{*}$} \\
\hline Nunca fumou & 225 & $\begin{array}{l}29,1 \\
63,4 \\
84,5\end{array}$ & 547 & $\begin{array}{l}70,9 \\
36,6 \\
15,5\end{array}$ & 0,000 \\
\hline Ex-fumante & 490 & 63,4 & 282 & 54,2 & \\
\hline Fuma atualmente & 676 & 87,5 & 96 & 12,5 & \\
\hline \multicolumn{6}{|l|}{ Consumo de bebidas alcoólicas $(\mathrm{n} \%)^{*}$} \\
\hline Não consome & 209 & 27,1 & 563 & 72,9 & 0,000 \\
\hline 0,2-54 gramas/dia & 354 & 45,8 & 418 & 54,2 & \\
\hline$>54$ gramas/dia & 676 & 87,5 & 96 & 12,5 & \\
\hline \multicolumn{6}{|l|}{ Classificação do PC ${ }^{1}(\mathrm{n} \%)^{*}$} \\
\hline Não & 344 & 44,5 & 428 & 55,5 & 0,000 \\
\hline Sim & 226 & 29,3 & 546 & 61,0 & \\
\hline \multicolumn{6}{|l|}{ Classificação do IMC² (n \%) } \\
\hline Normal & 266 & 34,4 & 506 & 65,6 & 0,057 \\
\hline Sobrepeso & 367 & 47,5 & 405 & 52,5 & \\
\hline Obeso & 379 & 49,1 & 393 & 50,9 & \\
\hline \multicolumn{6}{|l|}{ Tolerância à glicose ${ }^{3 \star}$ (n \%) } \\
\hline Normal & 246 & 31,8 & 526 & 68,2 & 0,012 \\
\hline Glicemia de jejum alterada (GJA) & 405 & 52,5 & 367 & 47,5 & \\
\hline Tolerância à glicose diminuída (TGD) & 343 & 44,5 & 429 & 55,5 & \\
\hline \multicolumn{6}{|l|}{ Classificação da PA4 (n \%) } \\
\hline Normal & 323 & 41,8 & 449 & 58,2 & 0,775 \\
\hline Alta & 341 & 44,2 & 431 & 55,8 & \\
\hline \multicolumn{6}{|l|}{ Dislipidemia $^{5 *}$ (n \%) } \\
\hline Não & 225 & 29,1 & 547 & 70,9 & 0,019 \\
\hline Sim & 350 & 45,3 & 422 & 54,7 & \\
\hline
\end{tabular}

DP: desvio-padrão; PC: perímetro de cintura; IMC: índice de massa corporal; ${ }^{*} p<0,05$, teste $X^{2}$.

${ }^{1}$ Pontos de corte segundo WHO/IASO/IOTF 2000: PC: $\operatorname{sim}=\geq 80 \mathrm{~cm}$ para mulheres; PC: $\operatorname{sim}=\geq 90 \mathrm{~cm}$ para homens.

2 Pontos de corte segundo WHO/IASO/IOTF 2000: IMC: $<23$ kg/m² eutrofia, 23 a 24,9kg/m² sobrepeso e $\geq 25 \mathrm{~kg} / \mathrm{m}^{2}$ obeso.

${ }^{3}$ Segundo critérios da WHO (2000) - Normoglicêmico $\geq 110 \mathrm{mg} / \mathrm{dL}$, e glicemia pós-carga de glicose < $140 \mathrm{mg} / \mathrm{dL}$; glicemia de jejum alterada: glicemia de jejum entre $110 \mathrm{mg} / \mathrm{dL}$ e $126 \mathrm{mg} / \mathrm{dL}$ com glicemia pós-carga de glicose < $140 \mathrm{mg} / \mathrm{dL}$; tolerância glicose diminuída: glicemia de jejum < $126 \mathrm{mg} / \mathrm{dL}$ e após sobrecarga entre $140 \mathrm{mg} / \mathrm{dL}$ e $200 \mathrm{mg} / \mathrm{dL}$.

${ }^{4}$ Segundo critérios da OMS: pressão arterial (PA) elevada = PA sistólica ou diastólica $\geq 140 / 90 \mathrm{mmHg}$.

${ }^{5}$ Segundo critérios da NCEP ou uso de medicamento: colesterol total $\geq 200 \mathrm{mg} / \mathrm{dL}, \mathrm{LDL}-\mathrm{c} \geq 130 \mathrm{mg} / \mathrm{dL}, \mathrm{HDL}-\mathrm{C} \leq 40 \mathrm{mg} / \mathrm{dL}$ ou triglicérides $\geq 150 \mathrm{mg} / \mathrm{dL}$. 
Tabela 2. Características gerais dos participantes segundo presença de obesidade generalizada, abdominal e generalizada na presença de abdominal

\begin{tabular}{|c|c|c|c|c|}
\hline & $\begin{array}{c}\text { Eutróficos } \\
(n=447)\end{array}$ & $\begin{array}{l}\text { Obesidade abdominal } \\
\qquad(n=54)\end{array}$ & $\begin{array}{l}\text { Obesidade geral } \\
\qquad(n=61)\end{array}$ & $\begin{array}{c}\text { Obesidade geral na } \\
\text { presença de abdominal } \\
(\mathrm{n}=\mathbf{2 1 0})\end{array}$ \\
\hline Gênero (\% homens/mulheres) ${ }^{\star}$ & $41,6 / 58,4$ & $18,5 / 81,5$ & $65,6 / 34,4$ & $44,3 / 55,7$ \\
\hline $\operatorname{IMC}\left(\mathrm{kg} / \mathrm{m}^{2}\right)^{1 \star \star}$ & $21,7(1,9)$ & $23,6(1,0)$ & $26,3(0,9)$ & $28,4(2,7)$ \\
\hline $\mathrm{PC}(\mathrm{cm})^{1 * *}$ & $75,4(6,5)$ & $85,1(5,0)$ & $83,1(5,3)$ & $92,1(7,8)$ \\
\hline Geração $(\% \text { issei/nisel })^{*}$ & $21,9 / 77,6$ & $24,1 / 75,9$ & $6,6 / 93,4$ & $11,5 / 88,5$ \\
\hline Idade $(\text { anos) })^{1 * \star}$ & $54,9(13,2)$ & $60,8(13,2)$ & $50,4(11,0)$ & $55,8(11,1)$ \\
\hline \multicolumn{5}{|l|}{ Alterações da homeostase glicêmica (\%)* } \\
\hline Normal & 40,3 & 42,6 & 34,4 & 19,0 \\
\hline Glicemia de jejum alterada (GJA) & 32,9 & 27,8 & 31,1 & 26,2 \\
\hline Tolerância à glicose diminuída (TGD) & 26,8 & 29,6 & 34,4 & 54,8 \\
\hline \multicolumn{5}{|l|}{ Pressão arterial (\%)* } \\
\hline Normal & 75,4 & 59,3 & 68,9 & 48,1 \\
\hline Alta & 24,6 & 40,7 & 31,1 & 51,1 \\
\hline HOMA-IR ${ }^{1 \star \star}$ & $0,30(0,6)$ & $0,55(0,61)$ & $0,57(0,50)$ & $0,91(0,60)$ \\
\hline
\end{tabular}

${ }^{1}$ Média e desvio-padrão (DP). ${ }^{*} P<0,05$, teste $X^{2} ;{ }^{* *} P<0,05$, ANOVA.

Tanto em homens quanto em mulheres nenhuma associação foi observada entre ingestão de gordura total e frações (com exceção do colesterol dietético) e obesidade abdominal ou obesidade generalizada na presença de abdominal. Além disso, em homens, nenhuma associação foi verificada entre ingestão de peixe, frango, carne vermelha e obesidade abdominal, bem como não houve associação entre obesidade generalizada na presença de abdominal e grupo das carnes vermelhas. A obesidade generalizada não se associou a nenhum fator dietético em ambos os gêneros (dados não apresentados).

\section{DISCUSSÃO}

No presente estudo, investigaram-se três diferentes padrões de distribuição de adiposidade corporal associados à ingestão de lipídios e alimentos embutidos ricos em gorduras. O primeiro padrão (obesidade generalizada) foi caracterizado pelo aumento da adiposidade geral, o segundo (obesidade abdominal) pelo acúmulo central e o terceiro com adiposidade excessiva generalizada inclusive na região abdominal. Esse último padrão foi investigado baseado na hipótese que poderia refletir pior perfil metabólico, assim como observado em outras populações (32). Em homens, nosso estudo sugere relação entre maior consumo de alimentos embutidos com a obesidade, principalmente a obesidade generalizada na presença da abdominal. Esses resultados foram também observados em estudos anteriores para a obesidade generalizada ou abdominal analisados separadamente $(7-8,14)$.
Embora a associação positiva entre consumo de ácido graxo oleico e obesidade abdominal observada no presente estudo, em homens, seja discordante de resultados de outros estudos em população ocidental (33), há uma possível explicação para essa associação: fontes dietéticas de ácido graxo oleico em nossa população de estudo coincidem com as fontes alimentares de gordura saturada (1). Os óleos vegetais são consumidos principalmente como frituras, o que combinaria com as fontes de gordura saturada. Logo, a estimativa de consumo desse nutriente reflete também o consumo de gordura saturada total da dieta. Outro estudo transversal de base populacional na população estadunidense comprovou que a alta ingestão de gordura saturada associava-se à obesidade abdominal (8). No entanto, analisando a natureza do estudo transversal, deve-se considerar também a possibilidade de o resultado ter sido ao acaso, bem como as próprias limitações dos dados de consumo alimentar.

Nesse estudo, outro nutriente que se associou à obesidade generalizada na presença de abdominal foi o colesterol. Em homens, observou-se que um consumo entre 233,83 e $920,63 \mathrm{mg} /$ dia de colesterol foi associado ao risco para obesidade generalizada com abdominal. As recomendações dietéticas para esses nutrientes são inferiores a $300 \mathrm{mg} /$ dia, sendo que os nipo-brasileiros chegaram a consumir até três vezes mais esse valor (1). Carrera e cols. (34), em estudo transversal de base populacional na população mexicana residente nos Estados Unidos (National Health and Nutrition 
Tabela 3. Odds ratios (intervalo com $95 \%$ de confiança) para obesidade abdominal $(n=329)^{1}$ e generalizada na presença de abdominal $(n=279)^{2}$ em homens segundo terços de consumo

\begin{tabular}{|c|c|c|c|c|c|}
\hline Fatores dietéticos & & $1^{0}$ terço & $2^{\circ}$ terço & $3^{0}$ terço & P-valor \\
\hline \multicolumn{6}{|l|}{ Obesidade abdominal ${ }^{1}$} \\
\hline \multirow[t]{7}{*}{ Ácido graxo oleico (g) } & n (normal/obeso) & $84 / 25$ & $71 / 39$ & $71 / 39$ & \\
\hline & Max-Min & $3,80-24,01$ & $24,02-32,47$ & $32,51-82,84$ & \\
\hline & Ajuste para idade & 1 & $0,99(0,54 ; 1,81)$ & $1,91(1,06 ; 3,47)^{*}$ & 0,039 \\
\hline & Modelo 1 & 1 & $0,92(0,48 ; 1,80)$ & $2,01(1,04 ; 3,89)^{\star}$ & 0,034 \\
\hline & Modelo 2 & 1 & $0,85(0,39 ; 1,85)$ & $1,98(1,02 ; 3,84)^{\star}$ & 0,039 \\
\hline & Modelo 3 & 1 & $1,54(0,47 ; 5,08)$ & $2,28(1,09 ; 4,80)^{\star}$ & 0,016 \\
\hline & Modelo 4 & 1 & $2,36(0,74 ; 7,56)$ & $6,80(1,91 ; 24,19)^{\star}$ & 0,012 \\
\hline \multirow[t]{7}{*}{ Embutidos (g) } & n (normal/obeso) & $78 / 26$ & $86 / 30$ & $62 / 47$ & \\
\hline & Max-Min & $0,00-6,70$ & $7,00-18,20$ & $18,60-95,30$ & \\
\hline & Ajuste para idade & 1 & $1,04(0,57 ; 1,92)$ & $2,25(1,25 ; 4,07)^{\star}$ & 0,007 \\
\hline & Modelo 1 & 1 & $0,96(0,49 ; 1,87)$ & $2,01(1,06 ; 3,85)^{\star}$ & 0,031 \\
\hline & Modelo 2 & 1 & $0,96(0,49 ; 1,87)$ & $2,00(1,01 ; 3,97)^{\star}$ & 0,047 \\
\hline & Modelo 3 & 1 & $0,81(0,37 ; 1,75)$ & $2,26(1,01 ; 3,97)^{\star}$ & 0,016 \\
\hline & Modelo 4 & 1 & $0,39(0,11 ; 1,34)$ & $2,97(0,89 ; 9,91)$ & 0,003 \\
\hline \multicolumn{6}{|c|}{ Obesidade geral na presença de abdominaR } \\
\hline \multirow[t]{6}{*}{ Colesterol (mg) } & n (normal/obeso) & $45 / 14$ & $66 / 33$ & $75 / 46$ & \\
\hline & Max-Min & $38,96-135,53$ & $136,48-206,90$ & $208,49-920,63$ & \\
\hline & Ajuste para idade & 1 & $1,55(0,81 ; 2,98)$ & $1,73(0,89 ; 3,37)$ & 0,247 \\
\hline & Modelo 1 & 1 & $1,87(0,87 ; 4,01)$ & $2,86(1,28 ; 6,40)^{\star}$ & 0,038 \\
\hline & Modelo 2 & 1 & $1,89(0,88 ; 4,09)$ & $2,99(1,32 ; 6,75)^{\star}$ & 0,032 \\
\hline & Modelo 3 & 1 & $1,62(0,65 ; 4,04)$ & $2,95(1,13 ; 7,72)^{\star}$ & 0,078 \\
\hline \multirow[t]{6}{*}{ Embutidos (g) } & n (normal/obeso) & $61 / 13$ & $61 / 22$ & $64 / 58$ & \\
\hline & Max-Min & $0,00-4,70$ & $5,00-14,00$ & $14,20-95,30$ & \\
\hline & Ajuste para idade & 1 & $1,66(0,77 ; 3,60)$ & $4,05(2,00 ; 8,19)^{\star}$ & 0,000 \\
\hline & Modelo 1 & 1 & $1,65(0,68 ; 3,98)$ & $4,31(1,90 ; 9,77)^{\star}$ & 0,001 \\
\hline & Modelo 2 & 1 & $1,63(0,67 ; 3,95)$ & $4,16(1,78 ; 9,72)^{\star}$ & 0,002 \\
\hline & Modelo 3 & 1 & $0,90(0,31 ; 2,57)$ & $2,97(1,13 ; 7,78)^{\star}$ & 0,009 \\
\hline \multirow[t]{6}{*}{ Aves (g) } & n (normal/obeso) & $48 / 16$ & $81 / 37$ & $57 / 40$ & \\
\hline & Max-Min & $0,00-2,20$ & $3,00-7,10$ & $9,00-135,00$ & \\
\hline & Ajuste para idade & 1 & $1,31(0,66 ; 2,62)$ & $1,97(0,97 ; 3,98)$ & 0,134 \\
\hline & Modelo 1 & 1 & $1,64(0,74 ; 3,63)$ & $2,47(1,08 ; 5,66)^{\star}$ & 0,094 \\
\hline & Modelo 2 & 1 & $1,64(0,74 ; 3,64)$ & $2,40(1,05 ; 5,51)^{\star}$ & 0,115 \\
\hline & Modelo 3 & 1 & $1,56(0,62 ; 3,94)$ & $2,07(0,79 ; 5,43)$ & 0,328 \\
\hline \multirow[t]{6}{*}{ Peixe e frutos do mar (g) } & $\mathrm{n}$ & $66 / 20$ & $58 / 33$ & $62 / 40$ & \\
\hline & Max-Min & $0,20-8,80$ & $9,00-20,20$ & $20,30-182,70$ & \\
\hline & Ajuste para idade & 1 & $1,94(0,99 ; 3,78)$ & $2,25(1,18 ; 4,30)^{\star}$ & 0,040 \\
\hline & Modelo 1 & 1 & $2,01(0,94 ; 4,31)$ & $2,56(1,20 ; 5,46)^{\star}$ & 0,047 \\
\hline & Modelo 2 & 1 & $1,96(0,92 ; 4,20)$ & $2,41(1,12 ; 5,18)^{\star}$ & 0,071 \\
\hline & Modelo 3 & 1 & $2,04(0,84 ; 4,97)$ & $2,29(0,94 ; 5,59)$ & 0,166 \\
\hline
\end{tabular}

${ }^{1}$ Obesidade abdominal: $P C \geq 80 \mathrm{~cm}$ para mulheres e $\geq 90 \mathrm{~cm}$ para homens (OMS/IASO/IOTF 2000).

${ }^{2}$ Obesidade abdominal: $P C \geq 80 \mathrm{~cm}$ para mulheres e $\geq 90 \mathrm{~cm}$ para homens e obesidade generalizada: IMC $\geq 25,0 \mathrm{~kg} / \mathrm{m}^{2}$ (WHO/IASO/IOTF 2000). ${ }^{*} \mathrm{P}<0.05$. Nutrientes ajustados pelas calorias totais (método residual); nutrientes e variáveis bioquímicas sofreram transformação logarítmica. Modelo 1: gênero, idade, geração, tabagismo, consumo de bebidas alcoólicas, hipertensão, dislipidemia, atividade física no trabalho. Modelo 2: modelo 1 + calorias totais (kcal). Modelo 3: modelo 2 + HOMA-IR. Modelo 4: modelo 3 + IMC. 
Examination Survey - NHANES 2001 e 2002), observaram padrão de ingestão aproximado ao da população nipo-brasileira também positivamente associado ao IMC e ao PC.

Em mulheres, no presente estudo houve associação inversa entre consumo de carnes vermelhas e obesidade geral com abdominal. Apesar de apenas um estudo anterior, transversal de base populacional, concordar com esse resultado (3), outras investigações longitudinais e uma metanálise não observaram essa associação $(7,8)$. Aquilo que se verificou no presente estudo é que a mediana de ingestão de carnes vermelhas no último tercil foi igual a 76,9 gramas (intervalo interquartil $=61,8$ 92,4 gramas), o que estaria de acordo com a recomendação dietética para população brasileira (35) e com a adequação proteica da dieta (1). Esse resultado relaciona-se provavelmente à melhor adequação nutricional da dieta entre mulheres nipo-brasileiras de acordo com o limite máximo de ingestão de carnes e alimentos proteicos recomendado.

No presente estudo, houve diferenças na associação entre padrões de distribuição de gordura corporal e ingestão de gorduras e alimentos cárneos segundo gênero. Algumas hipóteses podem explicar, pelo menos em parte, essas diferenças: 1) diferentes hábitos alimentares entre homens e mulheres nessa população. Na população de nipo-brasileiros de Bauru, SP, um estudo anterior observou maior ingestão de colesterol e gordura entre os homens (36). Possivelmente, os homens apresentam maior grau de ocidentalização e incorporação do estilo de vida ocidental em relação às mulheres nipo-brasileiras, o que poderia explicar diferenças quanto ao perfil antropométrico e frequência de morbidades em populações migrantes; 2 ) ocorrência de sub-relato do consumo de certos alimentos (como os alimentos não saudáveis) mais frequente em mulheres quando comparadas aos homens (37). No entanto, por essa razão, os modelos múltiplos foram adicionalmente ajustados pelas calorias totais para minimizar o impacto desse viés nos resultados; 3) diferente distribuição corporal da adiposidade em homens e mulheres. As mulheres podem apresentar maior quantidade de gordura subcutânea, enquanto os homens tendem ao acúmulo de gordura visceral (38). Em homens, há maior número de receptores $\alpha 2$-adrenorreceptores que aumentam a mobilização de ácidos graxos livres para o sistema venoso portal, favorecendo o acúmulo de tecido adiposo de localização visceral (39).
No entanto, a interpretação de nossos resultados exige cautela por seu delineamento ser transversal, considerando-se como uma das principais limitações a impossibilidade em identificar a relação temporal entre causa e efeito investigados. No entanto, essa limitação pode ter sido atenuada com a exclusão na presente análise dos indivíduos com diagnóstico prévio ou atual de AHG e DM ou em uso de hipoglicemiantes, visto que peso, PC, padrão alimentar e de atividades físicas podem se alterar com maior frequência devido à presença dessas morbidades (6).

Assim, os resultados apresentados sugerem que, em nipo-brasileiros, o maior consumo de alimentos provenientes de um padrão ocidentalizado, como o consumo de alimentos embutidos, está associado ao aumento da gordura corporal, principalmente à obesidade generalizada na presença de obesidade abdominal. Ressalta-se a importância de medidas estratégicas preventivas para a obesidade de acordo com o gênero, como o maior incentivo à alimentação saudável, sobretudo na população masculina.

Agradecimentos: à Fundação de Amparo à Pesquisa do Estado de São Paulo (Fapesp-01/11391-3R). Maria F. Cristofoletti recebeu, durante o período de doutorado, bolsa concedida pelo Conselho Nacional de Desenvolvimento Científico e Tecnológico $(\mathrm{CNPq}-140731 / 2007-08)$.

Declaração: os autores declaram não haver conflitos de interesse científico neste estudo.

\section{APÊNDICE}

Demais membros do Grupo de Estudos de Diabetes em Nipo-Brasileiros (Japanese-Brazilian Diabetes Study Group): Alcides Hirai, Amelia T. Hirai, Helena Harima, Katsumi Osiro, Mario Kikuchi, Luiza K. Matsumura, Renata Damião, Regina S. Moises, Newton de Barros Jr., Vânia D’Almeida (Universidade Federal de São Paulo, Departamento de Medicina Preventiva, São Paulo, SP, Brasil), Laércio J. Franco, Daniela S. Sartorelli (Faculdade de Medicina de Ribeirão Preto, Departamento de Medicina Preventiva, Universidade de São Paulo-USP, Ribeirão Preto, SP, Brasil), Nilce Tomita (Faculdade de Odontologia de Bauru, Departamento de Odontologia Social, USP, Bauru, SP, Brasil), Katsunori Wakisaka (Centro de Estudos de Nipo-Brasileiros, São Paulo, SP, Brasil); Rita Chaim (Universidade Sagrado Coração de Jesus, Departamento de Nutrição, Bauru, SP, Brasil). 


\section{REFERÊNCIAS}

1. World Health Organization (WHO). Diet, nutrition and the prevention of chronic diseases. WHO/FAO, Geneva: WHO; 2003. (Technical report series 916 )

2. Leite MLC, Nicolasi A. Lifestyle correlates of anthropometric estimates of body adiposity in an Italian middle-aged and elderly population: a covariance analysis. Int J Obes. 2006;30:926-34.

3. Garaulet M, Marin C, Pérez-Llamas F, Canteras M, Tebar FJ, Zamora S. Adiposity and dietary intake in cardiovascular risk in an obese population from a Mediterranean area. J Physiol Biochem. 2004;60:39-50.

4. Donadussi C, Oliveira AF, Fatel ECS, Dichi JB, Dichi I. Ingestão de lipídios na dieta e indicadores antropométricos de adiposidade em policiais militares. Rev Nutr. 2009;22:847-55.

5. Eck LH, Pascale RW, Klesges RC, White Ray JA, Klesges LM. Predictors of waist circumference change in healthy young adults. Int J Obes. 1995;19:765-9.

6. Koh-Banerjee P, Chu NF, Spiegelman D, Rosner B, Colditz G, Willett W, et al. Prospective study of the association of changes in dietary intake, physical activity, alcohol consumption, and smoking with 9-y gain in waist circumference among 16587 US men. Am J Clin Nutr. 2003;78:719-27.

7. Kahn HS, Tatham LM, Rodriguez C, Calle EE, Thun MJ, Heath CW Jr. Stable behaviors associated with adults' 10 -year change in body mass index and likehood of gain at the waist. Am J Public Health. 1997;87:747-54.

8. Maskarinec G, Takata Y, Pagano I, Carlin L, Goodman MT, Marchand $L L$, et al. Trends and dietary determinants of overweight and obesity in a multiethnic population. Obesity. 2006;14:717-26.

9. Fujimoto WY, Bergstrom RW, Boyko EJ, Leonetti DL, Newell-Morris LL, Wahl PW. Susceptibility to development of central adiposity among populations. Obes Res. 1995;3:S179-86.

10. Gimeno SGA, Ferreira SRG, Franco LJ, Hirai AT, Matsumara L, Moises RS. Prevalence and 7-year incidence of type II diabetes mellitus in a Japanese-Brazilian population: an alarming public health problem. Diabetologia. 2002;45:1635-8.

11. Fujimoto WY, Bergstrom RW, Boyko EJ, Chen KW, Kahn SE, Leonetti $\mathrm{DL}$, et al. Preventing diabetes-applying pathophysiological and epidemiological evidence. Br J Nutr. 2000;84:S173-S6.

12. Lerário DDG, Gimeno SG, Franco LJ, lunes M, Ferreira SRG, Grupo de Estudo de Diabetes na Comunidade Nipo-Brasileira, São Paulo, SP, Brasil. Excesso de peso e gordura abdominal para a síndrome metabólica em nipo-brasileiros. Rev Saúde Pública. 2002;36:4-11.

13. Rocha VZ, Libby P. The multiple facets of the fat tissue. Thyroid. 2008; 18:175-83.

14. Freire RD, Cardoso MA, Gimeno SG, Ferreira SR; Japanese-Brazilian Diabetes Study Group. Dietary fat is associated with metabolic syndrome in Japanese Brazilians. Diabetes Care. 2005;28:1779-85.

15. Damião R, Castro TG, Cardoso MA, Gimeno SGA, Ferreira SR. Japanese-Brazilian Diabetes Study Group. Dietary intakes associated with metabolic syndrome in a cohort of Japanese ancestry. Br J Nutr. 2006;96:532-8.

16. Salvo VLM, Cardoso MA, Barros Jr N, Ferreira SRG, Gimeno SGA. Consumo alimentar e doença macrovascular em nipo-brasileiros: um estudo transversal. Arq Bras Endocrinol Metab. 2009;53:865-73.

17. Sartorelli DS, Freire RD, Ferreira SRG, Cardoso MA; Japanese-Brazilian Diabetes Study Group. Dietary fiber and glucose tolerance in Japanese-Brazilians. Diabetes Care. 2005;28:2240-2.

18. Ridker PM, Cook N. Clinical usefulness of very high and very low levels of C-reactive protein across full range of Framinghan risk scores. Circulation. 2004;109:1955-9.

19. Chobanian AV, Bakris GL, Black HR, Cushman WC, Green LA, Izzo JL Jr, et al.; Joint National Committee on Prevention, Detection, Evaluation, and Treatment of High Blood Pressure. National Heart, Lung, and Blood Institute; National High Blood Pressure Education Program Coordinating Committee. Seventh report of the Joint National Committee on Prevention, Detection, Evaluation, and Treatment of High Blood Pressure. Hypertension. 2003;42:1206-52.
20. World Health Organization (WHO)/International Association for the Study of Obesity (IASO)/International Obesity Task Force (IOTF). The Asia-Pacific perspective: Redefining Obesity and its Treatment. Australia: Health Communications; 2000.

21. Cardoso MA, Kida AA, Tomita LY, Stocco PR. Reproducibility and validity of a food frequency questionnaire among women of Japanese ancestry living in Brazil. Nutr Res. 2001;21:725-33.

22. Cardoso MA, Stocco PR. Desenvolvimento de um questionário quantitativo de frequência alimentar em imigrantes japoneses e seus descendentes residentes em São Paulo, Brasil. Cad Saúde Pública. 2000;16:107-14.

23. Chiara VL, Sichieri R, Carvalho TSF. Teores de ácidos graxos trans de alguns alimentos consumidos no Rio de Janeiro. Rev Nutr. 2003;16:227-33.

24. Enig MG, Pallansch LA, Sampugna J, Keeney M. Fatty acid composition of the fat in selected food items with emphasis on trans components. J Am Oil Chem Soc. 1938;60:1788-94.

25. Bertolino CN, CastroTG, Sartorelli DS, Ferreira SRG, Cardoso MA; Grupo de Estudos de Diabetes na Comunidade Nipo-Brasileira. Influência do consumo alimentar de ácidos graxos trans no perfil de lipídios séricos em nipo-brasileiros de Bauru, São Paulo, Brasil. Cad Saúde Publica. 2006;22:357-64.

26. World Health Organization (WHO). Diabetes and Noncommunicable Disease Risk Factor Surveys. Geneva: WHO; 2000.

27. Alberti KGMM, Zimmet PZ for the WHO Consultation. Definition, Diagnosis and Classification of Diabetes Mellitus and its Complications. Part 1: Diagnosis and Classification of Diabetes Mellitus. Provisional Report of a WHO Consultation. Diabet Med. 1998;15:539-53.

28. Vieira JGH, Nishida SK, Lombardi N. Development of a monoclonal antibody-based immunofluorimetric assay for serum insulin and its comparison to a classical radioimmunoassay: implications of different specificities in the interpretation of clinical data. Braz J Med Biol Res. 1995;28:537-43.

29. Matthews DR, Hosker JP, Rundenski AS, Naylor BA, Treacher DF, Turner RC. Homestasis model assessment: insulin resistance and beta-cell function from fasting plasma glucose and insulin concentrations in men. Diabetologia. 1985;28:412-9.

30. Executive summary of the Third Report of the National Cholesterol Education Program (NCEP). Expert panel on detection, evaluation and treatment of high blood cholesterol in adults (adults treatment panel 3). JAMA. 2001;285:1486-97.

31. Willett WC, Stampfer M. Implications of total energy intake for epidemiologic analyses. In: Willett WC, editors. Nutritional Epidemiology. New York: Oxford University Press; 1998. p. 272-301.

32. Jacoby E, Goldstein J, López A, Núñez E, López T. Social class, family, and lifestyle factors associated with overweight and obesity among adults in Peruvian cities. Prev Med. 2003;37:396-405.

33. Romaguera D, Norat T, Mouw T, May AM, Bamia C, Slimani N, et al. Adherence to the Mediterranean diet is associated with lower abdominal adiposity in European men and women. J Nutr. 2010;139:1728-37.

34. Carrera PM, Xiang G, Tucker KL. A study of dietary patterns in the Mexican-American population and their association with obesity. J Am Diet Assoc. 2007;107:1735-42.

35. Ministério da Saúde. Guia alimentar para a população brasileira: promovendo a alimentação saudável. Brasília - DF: Ministério da Saúde; 2006.

36. Castro TG, Bertolino CN, Gimeno SGA, Ferreira SRG, Cardoso MA; Japanese-Brazilian Diabetes Study Group. Association of dietary fiber with temporal changes in serum cholesterol in Japanese-Brazilians. J Nutr Sci Vitaminol. 2006;52;203-10.

37. Maurer J,Taren DL, Teixeira PJ,Thompson CA, LohmanTG, Going $\mathrm{SB}$, et al. The psychosocial and behavioral characteristics related to energy misreporting. Nutr Rev. 2006;64:53-66.

38. Clarys JP, Martin AD, Drinkwater DT. The skinfold: myth and reality. J Sports Sci. 1987;5:3-33.

39. Lönnqvist $F$, Thörne A, Large V, Arner P. Sex differences in visceral fat lipolysis and metabolic complications of obesity. Arterioscler Thromb Vasc Biol. 1997;17:1472-80. 